\title{
The solar acceleration obtained by VLBI observations *
}

\author{
M. H. Xu ${ }^{1,2}$, G. L. Wang ${ }^{1}$, and M. Zhao ${ }^{1}$ \\ ${ }^{1}$ Shanghai Astronomical Observatory, Chinese Academy of Sciences, 200030 Shanghai, PR China \\ e-mail: [mhxu;wgl]@shao.ac.cn \\ 2 Graduate University of Chinese Academy of Sciences, 100049 Beijing, PR China
}

Received 14 May 2012 / Accepted 11 July 2012

\begin{abstract}
Aims. The three-dimensional acceleration vector of the solar system barycenter was estimated from VLBI delay observations. We also provide a first physical explanation for this acceleration vector.

Methods. Two methods for the acceleration estimation were developed in the analysis of the global astrometric/geodetic VLBI observations. One is to take the solar acceleration vector as a global parameter that is to be directly estimated. The second method is to obtain the acceleration by fitting the solar velocity variation time series produced from the VLBI data analysis.

Results. The results of these two methods are consistent with each other within their uncertainties. The acceleration vector derived from the first method is $(7.47 \pm 0.46,0.17 \pm 0.57,3.95 \pm 0.47) \mathrm{mm} \mathrm{s}^{-1} \mathrm{yr}^{-1}$ in the Galactic Cartesian coordinate system.

Conclusions. The Galactocentric component of the solar acceleration we obtained agrees well with the predicted value from the rotation movement of the Sun and has a high accuracy. The detected strong vertical component perpendicular to the Galactic plane may be important for the research of the Milky Way.
\end{abstract}

Key words. astrometry - reference systems - Galaxy: fundamental parameters - methods: data analysis

\section{Introduction}

Owing to the acceleration of the solar system barycenter (SSB), the original velocity of the International Celestial Reference System (ICRS, Arias 1995) is no longer a constant, but varies with time, which causes a systematic variation in the directions of the observed objects. This phenomenon is referred to as the secular aberration drift. Because of the relationship between this effect and the acceleration of the SSB, the acceleration can be directly determined from VLBI observations without any kinematic or dynamic models of the Milky Way.

The discussions of the secular aberration started in the late 20th century and have since attracted the attention of many astrometrists (see, e.g., Eubanks et al. 1995; Sovers et al. 1998; Perryman et al. 2001; Kovalevsky 2003; Kopeikin \& Makarov 2006; Titov 2010). Because the position accuracies of the observed radio sources are limited, it was hard to detect this effect from the VLBI observations, and it was always ignored in the routine VLBI data processing. Therefore, these studies were mainly theoretical discussions until the 2010s. Because VLBI observational accuracies have been improved, the data size has grown, and the models for data analysis have recently been optimized, it now becomes feasible to detect the effect of the secular aberration drift from VLBI observations. Titov et al. (2011) have analyzed 555 radio source position time series generated from the VLBI data analysis, from which they obtained the acceleration vector. The result was consistent with the value of the model prediction based upon two fundamental astronomical parameters, the solar distance to the Galactic center and the rotation speed around the Galactic center.

\footnotetext{
* Research supported by the National Science Foundation of China (Grant No. 10793032), and also by Shanghai Municipal Key Laboratory (Grant No. DZ22101).
}

Traditionally, much of the attention in the studies of the secular aberration was given to the Galactocentric component of the acceleration (Perryman et al. 2001; Titov et al. 2011), since it was generally believed that the acceleration component in the direction normal to the Galactic plane (hereafter vertical component or vertical acceleration) was too small to be detected. According to this scenario, the acceleration vector should point nearly to the Galactic center (Kopeikin \& Makarov 2006). The recent work of $\mathrm{Xu}$ et al. (2012), however, indicated that this vertical component was significant with an amplitude of $5.0 \pm 0.5 \mathrm{~mm} \mathrm{~s}^{-1} \mathrm{yr}^{-1}$. On the basis of this work, a new approach was developed and the parameters were re-analyzed with more recent data in two different ways in this paper. We furthermore endeavored to discuss this estimated acceleration of the SSB, especially the vertical component. The two methods will be briefly introduced in Sect. 2. The strategies for the VLBI data processing and the results will be given in Sect. 3. We finally discuss the results in Sect. 4.

\section{Two methods of estimation}

The direction of the observed radio source $\boldsymbol{K}$ was always treated as time-independent in the Barycentric Celestial Reference System (BCRS), which is a basic assumption of realizing the celestial reference system by radio sources (see, e.g., Feissel \& Mignard 1998; Ma 1998). But when included the effect of the secular aberration drift, the positions of the radio sources vary with time with a dipolar structure over the sky. Then at an arbitrary observing epoch $t$, the direction of the radio source is a function of the SSB's acceleration $\boldsymbol{a}$, given by

$$
\boldsymbol{K}_{t}=\boldsymbol{K}_{\mathbf{0}}+\frac{\left(\boldsymbol{K}_{\mathbf{0}} \times \boldsymbol{a}\right) \times \boldsymbol{K}_{\mathbf{0}}}{c}\left(t-t_{0}\right),
$$


where $\boldsymbol{K}_{\mathbf{0}}$ is the direction of the radio source in the BCRS at the reference epoch $t_{0}$ (e.g. J2000.0), and $c$ is the speed of light in a vacuum. Because the change of the SSB's velocity is very small with respect to the speed of light, the classical formula of the aberration is sufficient here. Because the rotation period of the Sun around the Galactic center is about 220 Myr (see Perryman 2009, Chap. 9), and the radial period equals $180 \mathrm{Myr}$, about three times of the period of the Sun's $z$ oscillation (Bailer-Jones 2009), the acceleration vector $\boldsymbol{a}$ could be treated as a constant during the about 30-year history of VLBI observations.

The direction of the radio source can also be expressed in another form as

$\boldsymbol{K}_{t}=\boldsymbol{K}_{\mathbf{0}}+\frac{\left(\boldsymbol{K}_{\mathbf{0}} \times \Delta \boldsymbol{v}_{t}\right) \times \boldsymbol{K}_{\mathbf{0}}}{c}$

Here, $\Delta v_{t}$ is the velocity variation of the SSB at the epoch $t$ and is given by

$\Delta \boldsymbol{v}_{t}=\boldsymbol{a}\left(t-t_{0}\right)+\delta \boldsymbol{v}$,

where $\delta v$ is the unmodeled fraction of the velocity variation.

According to the formula of the geometric delay given by the IERS 2010 conversion (Petit et al. 2010), the partial derivative of the VLBI geometric delay $\tau$ with respect to the instant direction $\boldsymbol{K}_{t}$, accurate up to terms of about $O\left(\left(\boldsymbol{V}_{\oplus}+\omega_{2}\right)^{2} / c^{2}\right)$, can be written as follows

$\frac{\partial \tau}{\partial \boldsymbol{K}_{t}}=-\frac{\boldsymbol{b}}{c+\boldsymbol{K}_{t}\left(\boldsymbol{V}_{\oplus}+\omega_{2}\right)}+\frac{\boldsymbol{K}_{t} \cdot \boldsymbol{b}}{c^{2}}\left(\boldsymbol{V}_{\oplus}+\omega_{2}\right)$,

where $b$ is the geocentric baseline vector, and $V_{\oplus}, \omega_{2}$ are the barycentric velocity of the geocenter and the geocentric velocity of the second receiver, respectively. Indeed, when calculating the value of this partial derivative, it is accurate enough to insert $\boldsymbol{K}_{\mathbf{0}}$ instead of $\boldsymbol{K}_{t}$ in the right hand side of Eq. (4).

From these equations, the partial derivatives of the VLBI geometric delay $\tau$ with respect to the acceleration and the velocity variation are given by the expressions

$\frac{\partial \tau}{\partial \boldsymbol{a}}=\frac{\partial \tau}{\partial \boldsymbol{K}_{t}} \cdot \frac{\partial \boldsymbol{K}_{t}}{\partial \boldsymbol{a}}$

$\frac{\partial \tau}{\partial \Delta \boldsymbol{v}_{t}}=\frac{\partial \tau}{\partial \boldsymbol{K}_{t}} \cdot \frac{\partial \boldsymbol{K}_{t}}{\partial \Delta \boldsymbol{v}_{t}}$.

Therefore, there are two approaches to estimate the acceleration vector. One is to take the solar acceleration vector as a global parameter based on Eq. (5), which is designated as a global solution. For the second method, two steps are currently used: (1) obtaining the time series of the velocity variations through Eq. (6) from VLBI observations; (2) fitting the acceleration based on Eq. (3) from this time series. We call this the time-series solution here. Since the latter method cannot effectively separate the velocity from other error sources, such as the influences of the position errors of the radio sources, the precision of the result obtained with this method cannot match that of the global solution. However, the time-series solution can clearly show the details and trends of the velocity variations of the SSB, and it can be taken as a validation for the first method as well. Additionally, since the velocity variation time series and radio source coordinates are not independent and thus cannot be obtained simultaneously without any other constraints, there are of course some differences between these two methods in the solution strategies, which will be discussed in detail in the next section.

\section{Data and results}

The data we used in our solutions were taken from the International VLBI Service for Geodesy and Astrometry (IVS, Schlüter \& Behrend 2007) data center. Sessions shorter than $18 \mathrm{~h}$ or with the longest baseline length shorter than $3000 \mathrm{~km}$ (small network) were excluded. In total, we selected 4632 sessions from April 1980 to October 2011. The data of the radio sources with less than four good observations in the whole data set were not used, and the data observed at the station TSUKUBA32 after the big earthquake in March 2011 were excluded as well. The elevation cut-off angle was set to $7^{\circ}$.

The CALC/SOLVE software developed and maintained by NASA Goddard Space Flight Center (Ma et al. 1990) was used for the VLBI data analysis. The USER_PARTIAL function, an external interface provided by this software, was used to integrate the estimation models of the acceleration and velocity variations into the VLBI data reduction. Each solution included the common parameterizations and specific parameterizations for an individual solution. Common parameters were divided into three groups:

- Global (over the entire data set): positions and velocities of the stations at the reference epoch; the axis offsets of the antennas; the coefficients of the diurnal, semi-diurnal, annual, and semi-annual tidal model (amplitude and phase) of 41 stations (Petrov \& Ma 2003); coefficients for the expansion into B-spline basis of the positions of three stations, HARS_085, PIETOWN, GILCREEK.

- Local (over each session): Earth orientation parameters, including the polar motion, UT1 and their changes along with the adjustments of two nutation parameters.

- Segmented (over 20 min-6 h): offsets and rates of clock function (1-h segment); coefficients of the linear spline that models zenith wet delay (20-min segment); coefficients of the piecewise function of the north and east troposphere gradients (6-h segment).

In the global solution, the acceleration vector $\boldsymbol{a}$ was taken as a global parameter. The positions of 39 special handling sources were considered as local parameters (Ma et al. 2009), while those of the other radio sources were treated as global parameters. No-net rotation constraints were imposed on the 295 ICRF2 defining sources with respect to their ICRF2 positions (Ma et al. 2009). In the time-series solution, the velocity variations were estimated once a year, while the strongest constraint was imposed on the radio source positions, which were not estimated and fixed to their ICRF2 coordinates.

For these two methods, no-net-rotation and no-nettranslation constraints on the adjustments of the station positions and velocities of 35 sites with respect to VTRF2008 (Böckmann et al. 2010) were applied to realize the Earth reference frame. The apriori models for geophysical effects and precession/nutation were based on the IERS 2003 conventions (McCarthy \& Petit 2004). NMF (Niell 1996) was chosen as the atmosphere mapping function, and we also applied the atmospheric pressure loading model (Petrov \& Boy 2004) and the thermal expansion model of the radio telescopes (Nothnagel 2009).

For all 4632 sessions, 3492 radio sources and approximately 7100000 group delay observables were analyzed. In the global solution, the overall post-fit weighted root mean square delay residual is $21.3 \mathrm{ps}$, and the chi-square is 0.98 . In the Galactic Cartesian coordinate system (Blaauw et al. 1960; Murray 1989), the acceleration vector in the three components is estimated to 
Table 1. The solar acceleration estimated from different data sets in the Galactic coordinate system (unit: $\mathrm{mm} \mathrm{s}^{-1} \mathrm{yr}^{-1}$ ).

\begin{tabular}{lccc}
\hline \hline Num. of Sess. & $X$ (galac. center) & $Y$ & $Z$ (galac. pole) \\
\hline 3474 & $7.44 \pm 0.53$ & $0.63 \pm 0.67$ & $3.27 \pm 0.54$ \\
3474 & $7.28 \pm 0.53$ & $-0.12 \pm 0.66$ & $3.93 \pm 0.53$ \\
3474 & $7.22 \pm 0.53$ & $0.06 \pm 0.66$ & $4.55 \pm 0.54$ \\
3474 & $7.86 \pm 0.53$ & $-0.41 \pm 0.67$ & $3.98 \pm 0.54$ \\
\hline
\end{tabular}

Notes. The four data groups are formed as follows: all sessions are sorted and numbered from 1 to 4632 in time order, then all sessions except the sessions whose numbers are $4 n+1$ are included in the first group, all sessions except the sessions whose numbers are $4 n+2$ are included in the second group and so on, where $n=0,1,2, \ldots, 1157$.

be $(7.47 \pm 0.46,0.17 \pm 0.57,3.95 \pm 0.47) \mathrm{mm} \mathrm{s}^{-1} \mathrm{yr}^{-1}$. To verify the reliability and stability of this result, the entire VLBI data set was divided into four groups according to the notes below Table 1, each of which was processed in the same way to estimate the acceleration. The results are listed in Table 1. The Galactocentric and vertical components show a good consistency between these four results, and the $Y$ components remain insignificant with respect to their formal errors.

In the time-series solution, the post-fit weighted root mean square residual delay is $21.9 \mathrm{ps}$, and the chi-square is 1.02 , both of which are slightly larger than those from the global solution. We recall here that the velocity variations were piecewise estimated per year. The velocity variation time series along with their error bars are illustrated in Fig. 1. Because of the poor precisions of the early VLBI observations, estimates of the velocity variations before 1985 were excluded. Applying the weighted linear fitting to the velocity variation time series, we obtained the three components of the acceleration $(9.10 \pm 1.74,0.56 \pm 1.60$, $4.53 \pm 1.88) \mathrm{mm} \mathrm{s}^{-1} \mathrm{yr}^{-1}$. These three components and their formal errors are all larger than those of the global solution, but the differences between these two methods are within their formal errors.

\section{Discussions and conclusions}

The main purpose of estimating the acceleration with the two different methods and from the different VLBI data groups is to check the stability and reliability of our results. From the velocity variation estimations shown in Fig. 1, we can clearly see the trends caused by the acceleration, and the considerable biases, $\delta \boldsymbol{v}$, caused by some time-dependent noises or the realistic nonlinear variations of the SSB's velocity, which can be studied and identified in the near future with a longer VLBI observational span. These biases cause the large formal errors of the acceleration fitted from the velocity variations. The velocity variations were also estimated in different time spans, such as every half year and every two years, and the trends obtained are invariant throughout. The two methods show a consistent estimate of the solar acceleration. Because of its higher accuracy, the acceleration vector based on the global solution $(7.47 \pm 0.46,0.17 \pm 0.57$, $3.95 \pm 0.47) \mathrm{mm} \mathrm{s}^{-1} \mathrm{yr}^{-1}$ in the Galactic coordinate system, is recommended as the formal estimate in this work. Moreover, if we take this result as an initial value of the acceleration, and estimate its correction according to the global solution, it becomes insignificant with respect to its formal error as $(0.04 \pm 0.46$, $0.08 \pm 0.57,-0.02 \pm 0.47) \mathrm{mm} \mathrm{s}^{-1} \mathrm{yr}^{-1}$. This confirms that our estimation statistically converges.

As previously stated, Titov et al. (2011) have also obtained the acceleration with VLBI observations, which is listed in
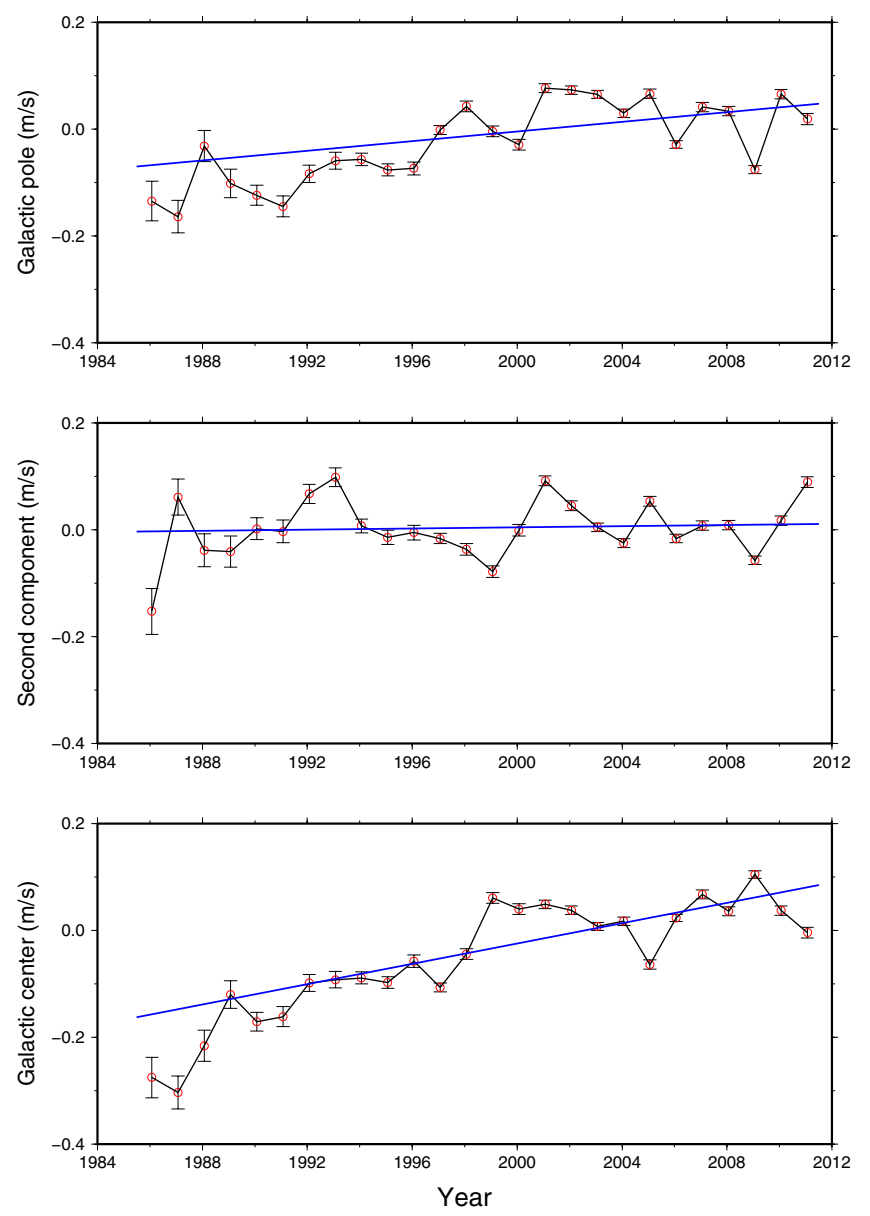

Fig. 1. Velocity time-series and its linear trend obtained from the timeseries solution.

Table 2. Comparison of the acceleration estimates (unit: $\mathrm{mm} \mathrm{s}^{-1} \mathrm{yr}^{-1}$ ).

\begin{tabular}{lccc}
\hline \hline & $X$ (galac. center) & $Y$ & $Z$ (galac. pole) \\
\hline TLG11 solution & $9.19 \pm 2.11$ & $1.01 \pm 1.95$ & $1.10 \pm 1.77$ \\
Global solution & $7.47 \pm 0.46$ & $0.17 \pm 0.57$ & $3.95 \pm 0.47$ \\
Time-series solution & $9.10 \pm 1.74$ & $0.56 \pm 1.60$ & $4.53 \pm 1.88$ \\
\hline
\end{tabular}

Table 2 with the notation TLG11 after being transformed and represented in the same unit as our result. Comparing the TLG11 and our results listed in Table 2, it is quite obvious that the global solution has the potential to obtain the result about four times better in formal error than the other two post-fit methods. The Galactocentric components of these three approaches coincide with each other as do the $Y$ components. There is, however, a large difference in the vertical component between our two methods and the TLG11 solution.

There are many papers that are directly or indirectly concerned with the Galactocentric component of the solar acceleration. Since the 1980 s, researches have shown that the solar distance to the Galactic center $R_{0}$ still lies in a broad range from $7.5-8.5 \mathrm{kpc}$, and $8.2 \mathrm{kpc}$ is often recommended as the best estimate (see Perryman 2009, Chap. 9). The rotation speed of the local standard of rest (LSR) varies between $195 \mathrm{~km} \mathrm{~s}^{-1}$ and $255 \mathrm{~km} \mathrm{~s}^{-1}$, hence the Galactocentric acceleration can be anything between $4.7-8.1 \mathrm{~mm} \mathrm{~s}^{-1} \mathrm{yr}^{-1}$ if $R_{0}=$ $8.2 \mathrm{kpc}$ is assumed. Recently, upon the Bar and Spiral Structure Legacy (BeSSeL) survey, Brunthaler et al. (2011) gave the best 
values of $R_{0}=8.3 \pm 0.23 \mathrm{kpc}$ and $\theta_{0}=239$ or $246 \pm 7 \mathrm{~km} \mathrm{~s}^{-1}$, for solar motions of $V_{\odot}=12.23$ and $5.25 \mathrm{~km} \mathrm{~s}^{-1}$, respectively, and suggested that the corresponding recommended values of the IAU1985 (Kerr \& Lyndenbell 1986) should be revised. Adopting values of the solar distance and velocity from this latest research, the Galactocentric acceleration can be derived with an amplitude of $7.7 \pm 0.48 \mathrm{~mm} \mathrm{~s}^{-1} \mathrm{yr}^{-1}$, which is quite close to our result. The value of the $Y$ component of the acceleration is not notable in our estimation, which agrees with the commonly assumed movement of the Sun in the Galactic plane.

The vertical component, however, has almost the same magnitude as the Galactocentric component and no other direct observation is available at present. Yet, the vertical component of the SSB's acceleration can likely be explained by three potential possibilities, which we present here in a brief first assessment.

The first possibility is that a planet $X$ of the Sun as yet undetected may exist in addition to those already known. As a result of the successful prediction of the location of Neptune through the residuals in the longitude of Uranus, the astronomical community has started to search for the evidence of undiscovered massive planets with a mass approximately from that of Mars to typical values of brown dwarfs $\left(m_{X} \approx 80 m_{\text {Jup }}\right.$ ) (see, e.g., Rawlins 1970; Seidelmann 1971; Hogg et al. 1991; Matese \& Whitmire 2011; Iorio 2012). The latest research by Iorio (2012) argued that a planet $X$ with $70 \%$ of the mass of the Earth cannot exist at a distance less than about 250-450 AU, and the minimum distance is 150-200 AU for mass of Mars, which is 3500-4500 AU for $4 m_{\mathrm{Jup}}$. Unfortunately, a planet $X$ constrained by these conditions cannot exert such a fast acceleration on the SSB, whose contribution to the acceleration is one magnitude smaller than the vertical acceleration.

The hypothesis of a companion star orbiting the Sun should offer more room for explaining the vertical component of the acceleration, and indeed, normally stars are members of double star systems or multiple systems (Donnison 1984). Harrison (1977) firstly suggested that the Sun has an undetected companion star as an explanation of the decrease in the period of a small group of pulsars. Later this was discussed in more detail by Cowling (1983) and Thornburg (1985). However, these studies could only constrain the SSB's acceleration to $\sim 32 \mathrm{~mm} \mathrm{~s}^{-1} \mathrm{yr}^{-1}$, which is much higher than the value of our vertical component, $3.95 \mathrm{~mm} \mathrm{~s}^{-1} \mathrm{yr}^{-1}$. Recently, Zakamska \& Tremaine (2005) used the timing data of millisecond pulsars, pulsars in binary and pulsating white dwarfs to determine the acceleration. They used the theoretical Galactocentric acceleration $a_{\mathrm{Gal}, \odot}$ to correct the observed period derivatives before the data analysis, and then they constrained the SSB's remaining acceleration on the upper limit of $4.73 \mathrm{~mm} \mathrm{~s}^{-1} \mathrm{yr}^{-1}$, comparable with our vertical component. So the vertical component we obtained may provide a direct observational evidence of the existence of the solar companion.

According to the third explanation, it is also very likely that this component arises from the oscillation motion of the Sun. The Sun oscillates perpendicular to the Galactic plane when it travels in the Galaxy (see, e.g., Gould \& Vandervoort 1972; Bahcall \& Bahcall 1985; Shuter \& Klatt 1986; Binney \& Tremaine 2008). However, the direction and magnitude of the vertical acceleration are both questionable in this explanation. Because the Sun is located in the north of the Galactic plane (see, e.g., Humphreys \& Larsen 1995; Perryman 2009, Chap. 9), the vertical acceleration should point to the south of the Galactic pole, which is the inverse direction of our result. Moreover, the Sun is considered to be near the plane and the vertical component is supposed to be one order of magnitude smaller than the Galactocentric component (Kopeikin \& Makarov 2006). Two points need to be emphasized here for these two questions. First, this plane defined by the $\mathrm{H} 1$ disk itself has a probable error of $0.12^{\circ}$ (Blaauw et al. 1960) and this angular discrepancy corresponds to $17 \mathrm{pc}$ distance at the place of $R=8.3 \mathrm{kpc}$. Secondly, the basic principle to define the Galactic plane according to $\mathrm{H} 1$ disk is that the Galactic neutral hydrogen is confined to a thin and flat layer in the inner regions of the Galaxy $R<7 \mathrm{kpc}$ and is systematically distorted in the outer parts according to the studies of 21-cm radiation (Kerr et al. 1957; Westerhout 1957; Gum et al. 1960). On the contrary, this would imply that the present Galactic plane could not represent the dynamical plane near the Sun $R>7 \mathrm{kpc}$, where the neutral hydrogen has already been a distorted distribution. Consequently, although this vertical acceleration could be determined by models for the Galaxy to have an amplitude of less than $0.79 \mathrm{~mm} \mathrm{~s}^{-1} \mathrm{yr}^{-1}$ (see Gould \& Vandervoort 1972; Cox 2000), the Galactic plane and models remain quite uncertain. An example of these is the mass model presented by Dehnen \& Binney (1998) and McMillan (2011). If this vertical acceleration is caused by the solar oscillation in the Galaxy, this would mean that the dynamical plane near the Sun is tilted with respect to the Galactic plane in a dramatically angle and the mass model for the Galaxy may need to be investigated.

Because of the insufficient knowledge of the Galaxy, much more work is needed to explain the vertical acceleration clearly. At the present time, however, we tend to believe that the main cause of this significant vertical acceleration is that the Sun has an undetected companion star. If that is the case, it would be beneficial to constrain the direction and the mass of this companion. This vertical acceleration is of great theoretical importance, since it is for the first time obtained directly from observations with high accuracy. Apparently, this vertical acceleration will provide another meaningful parameter for the research of the Milky Way. The future space missions of sub-milliarcsecond astrometry (SIM Lite and Gaia), which will establish a new referenceframe and then allow a direct detection of the acceleration of the SSB (Johnston 2009), are also expected to verify the result from VLBI observations.

Acknowledgements. The authors greatly appreciate the detailed advice from the anonymous referee. The authors also would like to thank Jin Wenjing for the fruitful discussions of this work and John M. Gipson from GSFC for his kind help with the USER_PARTIAL function.

\section{References}

Arias, E. F., Charlot, P., Feissel, M., et al. 1995, A\&A, 303, 604

Bahcall, J. N., \& Bahcall, S. 1985, Nature, 316, 706

Bailer-Jones, C. A. L. 2009, IJAsB, 8, 213

Binney, J., \& Tremaine, S. 2008, Galactic Dynamics, second edition (Princeton: Princeton University Press)

Blaauw, A., Gum, C. S., Pawsey, J. L., \& Westerhout, G. 1960, MNRAS, 121, 123

Böckmann, S., Artz, T., \& Nothnagel, A. 2010, J. Geod, 84, 201

Brunthaler, A., Reid, M. J., Menten, K. M., et al. 2011, Astron. Nachr., 332, 461 Cowling, S. A. 1983, MNRAS, 204, 1237

Cox, A. N. 2000, Allen's astrophysical quantities (New York: AIP Press)

Dehnen, W., \& Binney, J. 1998, MNRAS, 294, 429

Donnison, J. 1984, EM\&P, 30, 107

Eubanks, T. M., Matsakis, D. N., Josties, F. J., et al. 1995, in Astronomical and Astrophysical Objectives of Sub-Milliarcsecond Optical Astrometry, eds. E. Hog, \& P. K. Seidelmann, IAU Symp., 166, 283

Feissel, M., \& Mignard, F. 1998, A\&A, 331, L33

Gould, E., \& Vandervoort, P. O. 1972, AJ, 77, 360

Gum, C., Kerr, F., \& Westerhout, G. 1960, MNRAS, 121, 132

Harrison, E. R. 1977, Nature, 270, 324

Hogg, D., Quinlan, G., \& Tremaine, S. 1991, AJ, 101, 2274

Humphreys, R., \& Larsen, J. 1995, AJ, 110, 2183 
M. H. Xu et al.: The solar acceleration obtained by VLBI observations

Iorio, L. 2012, CeMDA, 112, 117

Johnston, K., Wehrle A. E., Makarov, V., et al. 2009, in Astronomy, 2010, astr2010: The Astronomy and Astrophsics Decadal survey, 143

Kerr, F., \& Lyndenbell, D. 1986, MNRAS, 221, 1023

Kerr, F., Hindman, J., \& Carpenter, M. 1957, Nature, 180, 677

Kopeikin, S., \& Makarov, V. 2006, AJ, 131, 1471

Kovalevsky, J. 2003, A\&A, 404, 743

Ma, C., Sauber, J. M., Clark, T. A., et al. 1990, J. Geophys. Res., 95, 21991

Ma, C., Arias, E. F., Eubanks, T. M., et al. 1998, AJ, 116, 516

Ma, C., Arias, E. F., Bianco, G., et al. 2009, International Earth Rotation and Reference Systems Service (IERS), Technical Note 35, Frankfurt am Main 2010: Verlag des Bundesamts für Kartographie und Geodäsie

Matese, J. J., \& Whitmire, D. P. 2011, ICARUS, 211, 926

McCarthy, D. D., \& Petit, G. 2004, IERS Technical Note 32, Frankfurt am Main 2004: Verlag des Bundesamts für Kartographie und Geodäsie

McMillan P. J. 2011, MNRAS, 414, 2446

Murray, C. 1989, A\&A, 218, 325

Niell, A. 1996, J. Geophys. R., 101, 3227

Nothnagel, A. 2009, J. Geod, 83, 787
Perryman, M. 2009, Astronomical Applications of Astrometry: Ten Years of Exploitation of the Hipparcos Satellite Data (Cambridge: Cambridge University Press)

Perryman, M., de Boer, K., Gilmore, G., et al. 2001, A\&A, 369, 339

Petit, G., \& Luzum, B. 2010, IERS Technical Note 36, Frankfurt am Main 2010: Verlag des Bundesamts für Kartographie und Geodäsie

Petrov, L., \& Boy, J. 2004, J. Geophys. R., 109, 345

Petrov, L., \& Ma, C. 2003, J. Geophys. R., 108, 2190

Rawlins, D. 1970, AJ, 75, 856

Schlüter, W., \& Behrend, D. 2007, J. Geod., 81, 379

Seidelmann, P. K. 1971, AJ, 76, 740

Shuter, W. L. H., \& Klatt, C. 1986, ApJ, 301, 471

Sovers, O., Fanselow, J., \& Jacobs, C. 1998, Rev. Mod. Phys., 70, 1393

Thornburg, J. 1985, MNRAS, 213, P27

Titov, O. 2010, MNRAS, 407, L46

Titov, O., Lambert, S. B., \& Gontier, A.-M. 2011, A\&A, 529, A91

Westerhout, G. 1957, Bull. Astron. Inst. Netherlands, 13, 201

Xu, M., Wang, G., \& Zhao, M. 2012, Sci. China G: Phys. Astron., 55, 329

Zakamska, N., \& Tremaine, S. 2005, AJ, 130, 1939 